one case there were seven entrance wounds and five exit wounds, only two ballbearings being recoved from the body. One had lodged in the anterior abdominal wall, and the other in the body of the fifth lumbar yertebra.

In a second case a ballbearing had entered through the ninth rib in the mid-axillary line and, flying through the dome of the diaphragm and the liver, had torn across the right ventricle to be recovered from the left chest. A second ballbearing had entered through the right buttock, had shattered the ilium, and was recovered from the thickness of the left acetabulum (fig 3). These missiles were about $6 \mathrm{~mm}$ in diameter. The one in the acetabulum was extremely difficult to identify owing to the thickness of the acetabulum (about $7 \mathrm{~mm}$ ). $X$-ray pictures are essential in such cases, and usually the bomb squad require every piece of metal to be recovered. Furthermore, such missiles may be carried by the blood stream from one part of the body to another (fig 4).

In shooting there is generally some aim taken and some expected direction of fire, but in terrorist bomb explosions the direction of flight of objects such as ballbearings is random.

\title{
IgA Deficiency in one of identical twins
}

\author{
R M LEWKONIA， DOUGLAS GAIRDNER，W F DOE
}

British Medical fournal, 1976, 1, 311-313

\section{Summary}

A disease resembling childhood coeliac disease occurred in one of identical twins. When the twins were investigated at the age of 23 the initial diagnosis could not be substantiated but the twin who had been ill had selective IgA deficiency. Differences dating from early infancy may have been a result of the discordance for IgA deficiency. Thus in some patients environmental influences may be important in either the initiation or the perpetuation of the deficiency.

\section{Introduction}

Several gastrointestinal disorders, including gluten-sensitive enteropathy and some less clearly defined malabsorption syndromes, sometimes occur in association with IgA deficiency. ${ }^{12}$ Either a causal relationship or a common pathogenesis might account for the association. ${ }^{3}$ We report here the occurrence of selective IgA deficiency in one of identical twins associated with an illness which in childhood superficially resembled coeliac disease. As the twins were identical in almost all other respects the "coeliac-like" syndrome was probably a consequence rather than simply a concomitant of the IgA deficiency.

\section{Case report}

Girl twins both weighed $3.46 \mathrm{~kg}$ at birth in 1951 . Shortly after weaning to cereals at three months one twin (twin 1) started to vomit after feeds and thereafter she thrived less well than her sister. At six months both twins had an acute diarrhoeal illness; this was mild in twin 1 but much more severe in twin 2, who was admitted to hospital.

The failure of twin 1 to thrive continued and she was first seen when aged 33 months with a nine-month history of persistent vomiting after

Nuffield Unit of Medical Genetics, Department of Medicine, University of Liverpool, Liverpool

R M LEWKONIA, MRCP, DCH, Nuffield research fellow (present address: Hammersmith Hospital, London)

Department of Paediatrics, Addenbrooke's Hospital, Cambridge DOUGLAS GAIRDNER, DM, FRCP, consultant paediatrician

Department of Medicine, Royal Postgraduate Medical School, Hammersmith Hospital, London

W F DOE, MSC, MRCP, lecturer (present address: department of immunology, Scripps Clinic and Research Foundation, La Jolla, California)

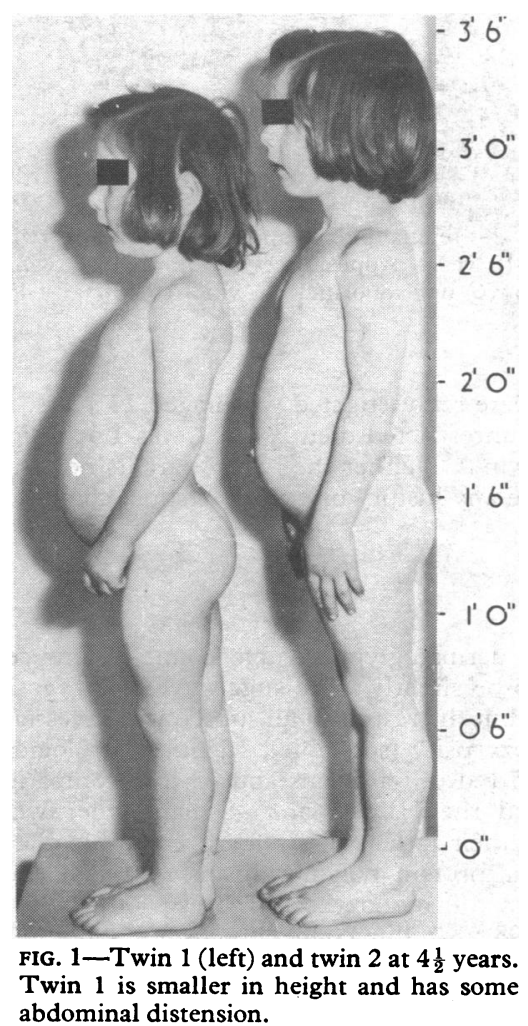

feeds, intermittent abdominal distension, and loose offensive stools There was a severe hypochromic anaemia and initial findings were consistent with the clinical diagnosis of coeliac disease. A gluten-free diet was advised, but her bowel symptoms had improved before this was started and her parents discontinued the diet after eight months. Throughout childhood twin 1 lagged behind in growth (fig 1): at eight years she had a radiological bone age of 6 years and was $4.5 \mathrm{~kg}$ lighter and $8 \mathrm{~cm}$ shorter than her twin. Twin 1 had repeated sore throats and tonsillectomy was performed when she was 10 . When she was 13 twin 1 complained of occasional cramp-like pain, but diarrhoea was no longer a symptom. The weight difference was now $6.5 \mathrm{~kg}$, and twin 1, unlike her sister, showed no evidence of secondary sex characteristics. Jejunal biopsy performed at 13 years showed partial villous atrophy with round cell infiltration of the lamina propria and intraepithelial lymphocytes in the mucosa of twin 1 (fig 2). There was no evidence of giardiasis. The biopsy appearances in twin 2 were normal. Twin 1 resumed gluten restriction for a further 18 months, during which the occasional abdominal pain resolved but the growth disparity increased. Menarche occurred at 15 years 6 months in twin 1,18 months later than in twin 2 . 

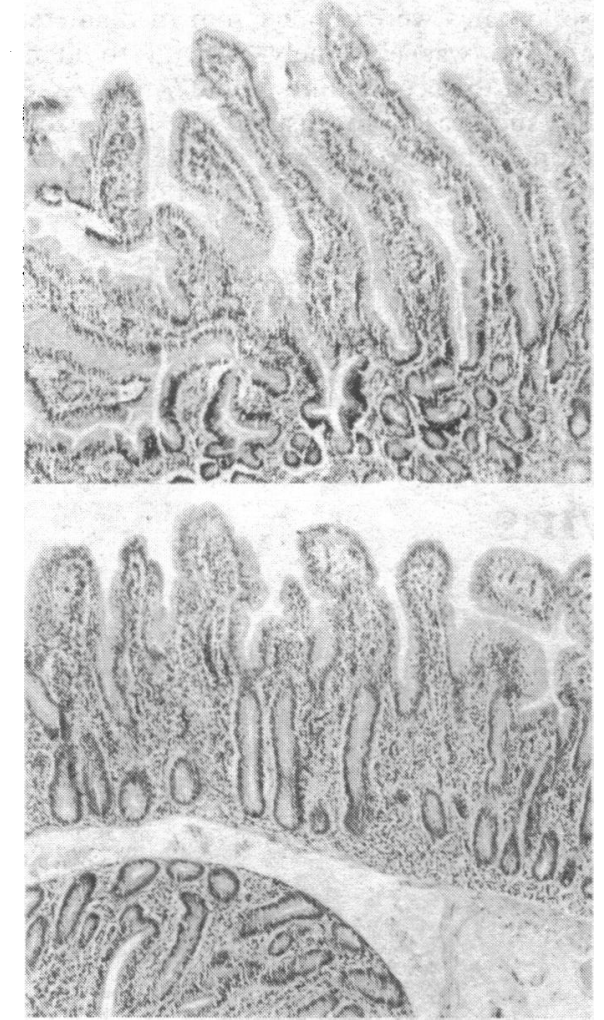

FIG 2-Jejunal biopsy appearances in both twins at 13 years. Appearances are normal in twin 2 (above) but abnormal in twin 1 (below). $(\times 45$.)

The twins were reinvestigated when aged 23 years. Both were well and taking an unrestricted diet. Twin 1 was $1.5 \mathrm{~kg}$ lighter and $2 \mathrm{~cm}$ shorter than twin 2. Neither the twins' parents nor any of the twins' five sisters gave any history of gastrointestinal complaints.

\section{INVESTIGATIONS}

Zygosity-A dermatoglyphic ridge count difference of 31 ridges was thought to be slightly more suggestive of dizygosity rather than monozygosity, but the results of all subsequent investigations strongly favoured monozygosity (see table). Identical serological results were obtained for 25 red cell antigens and 23 histocompatibility antigens. Both twins had the HLA-1 and -8 haplotype, which they had inherited from their father. Tests for five red blood cell isoenzymes and two plasma protein polymorphisms also gave identical results. The probability of monozygosity, based on these findings, was greater than $0.98 .^{4}$ Satellite counts on chromosome preparations were virtually identical for the twins and both had the same complex type of dental malocclusion, which required orthodontic treatment.

Immunoglobulins-Serum immunoglobulin concentrations were measured by single radial immunodiffusion and the results are shown in the table. Twin 1 had selective IgA deficiency with a serum concentration of $0.02 \mathrm{~g} / 1$; this immunoglobulin could not be shown by immunoelectrophoresis (fig 3). IgA secretory piece was shown in the saliva of both twins. Serum specimens from both parents and four of the five sisters contained normal quantities of IgA. Only saliva was obtainable from the fifth sister and IgA was present at the normal

Serum immunoglobulin concentrations, tissue typing, and red cell grouping in both twins

\begin{tabular}{|c|c|c|c|c|c|c|}
\hline & \multicolumn{4}{|c|}{$\underset{(\mathbf{g} / \mathbf{l})}{\operatorname{Serum}} \underset{\mathrm{immuno}}{ }$} & \multirow{2}{*}{$\begin{array}{c}\text { Histocompatibility } \\
\text { antigens }\end{array}$} & \multirow{2}{*}{$\begin{array}{l}\text { Red blood cell } \\
\text { groupings }\end{array}$} \\
\hline & IgG & IgM & IgA & IgD & & \\
\hline $\begin{array}{l}\text { Twin 1 } \\
\text { Twin 2 }\end{array}$ & $\begin{array}{l}12 \cdot 85 \\
14.57\end{array}$ & $\begin{array}{l}1.28 \\
0.89\end{array}$ & $\begin{array}{l}0.02 \\
1.2\end{array}$ & ND & \}$_{\text {HLA-10 }}^{\text {HLA-1, HLA-8, }}$ & $\begin{array}{l}\mathrm{A}_{1}, \mathrm{MNs}, \mathrm{cDE} / \mathrm{cDE}, \\
\mathbf{P}_{1} \mathbf{L u}\left(\mathbf{a}^{-}\right), \mathbf{K}^{-} \mathbf{K}^{+}, \\
\mathbf{K} \mathbf{p}\left(\mathbf{a}^{-}\right), \mathbf{F y} \\
\left(\mathbf{a}^{+} \mathbf{b}^{-}\right), \mathrm{Jk}\left(\mathbf{a}^{-} \mathbf{b}^{+}\right) \\
\operatorname{Le}\left(\mathbf{a}^{-}\right)\end{array}$ \\
\hline
\end{tabular}

$\mathrm{ND}=$ Not detected

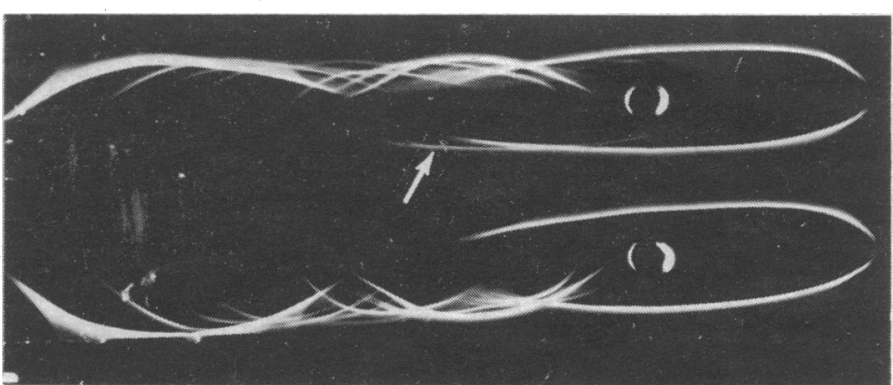

FIG 3-Immunoelectrophoresis of twins' sera. Upper well contains twin 2's serum; lower well contains twin 1's serum. Upper and lower troughs: antiwhole human serum; central trough: anti-IgG and anti-IgA. Normal IgA arc is arrowed.

concentration. IgD was not detectable by radial immunodiffusion in the sera of either twin but was present in both parents' sera.

fejunal biopsies-Jejunal biopsies at 23 years showed normal appearances in twin 2 and a minor degree of partial villous atrophy in twin 1 , which was less pronounced than in the childhood biopsy. The appearances were not those of coeliac disease. Immunofluorescence studies showed a diminution in IgA-staining cells and a relative increase in IgM-staining cells in twin 1 compared with the normal appearances of twin 2 .

\section{Discussion}

The special interest of this case is the fact that in a pair of twins in whom monozygosity was proved with near certainty only one of the pair had selective IgA deficiency, which had been associated in childhood with a syndrome resembling coeliac disease. Although the clinical picture in twin 1 at first strongly suggested coeliac disease, that diagnosis was not sustained, for there was neither a clear-cut clinical response to a gluten-free diet nor a relapse when gluten was reintroduced. The jejunal biopsy of the affected twin was clearly abnormal when examined at the age of 13 , but the degree of villous atrophy was not as complete as is seen in coeliac disease.

The occurrence of selective IgA deficiency and a childhood "coeliac-like" syndrome associated with differing growth rates would be readily explicable if the twins were dizygotic. Although the configuration of the placenta is not known, all the serological and biochemical evidence overwhelmingly favoured monozygosity.

The immunoglobulin levels in these twins were first measured when they were 23 years old. But the early history of failure to thrive, the growth retardation, and the recurrent sore throats necessitating tonsillectomy, all suggest that twin 1 probably had selective IgA deficiency from infancy. IgD was not detected in the serum of either twin but the significance of this finding is obscure. IgD has been reported to be detectable less often in patients with coeliac disease than in controls. ${ }^{5}$

About 1 in 700 of the population have selective IgA deficiency, ${ }^{6}$ and most of these people are healthy. The factors that determine the development of associated gastrointestinal, respiratory, or other illnesses are obscure. The twins in this case almost certainly had identical sets of genes, including those coding for immune responses and for the HLA-1 and HLA-8 antigens, which have been associated with the true coeliac disease. ${ }^{7}$ IgA was present in subnormal amounts in the serum and jejunal biopsy specimen of twin 1 so that both twins must have possessed the genotype necessary to produce IgA. Some regulatory mechanism must have been at fault in the twin with the deficiency. Recent work suggests two possible mechanisms that could produce a selective immunoglobulin deficiency. There might be a fault in the sequential fetal differentiation of classes of B lymphocytes before contact with exogenous antigens. ${ }^{8}$ Alternatively, there might be inhibition of a class of B lymphocytes by specific suppressor $\mathrm{T}$ lymphocytes, ${ }^{9}$ perhaps induced by infection. ${ }^{10}$ The latter seems a more likely mechanism in our 
case because there was a deficiency rather than an absence of serum IgA.

In seeking for some environmental factor that might explain the discordance for IgA deficiency in these twins the only impressive difference in their early life was the episode of gastroenteritis at six months, which was far more severe in the subsequently healthy twin. Work in germ-free animals ${ }^{11}$ suggests that stimulation by micro-organisms is important in promoting IgA synthesis by the gut. By analogy, the genotype common to these twins might have required a particularly strong stimulus to switch-on IgA synthesis and the episode of gastroenteritis might have occurred at a time critical for the establishment of endogenous IgA production. Whatever the cause of the discordance for IgA deficiency, however, it seems likely that this defect and an associated malabsorption accounted for the slower growth and the childhood illness of one of these twins.

Selective IgA deficiency is sometimes familial, but the reported patterns of familial involvement are variable and the mode of inheritance is not well defined. ${ }^{1:}$ Its occurrence in one of identical twins suggests that environmental influence is important in either the initiation or the perpetuation of the deficiency in some people.

We thank Professor Sir Cyril Clarke and Professor C C Booth for their interest and advice. Dermatoglyphic ridge count was performed by the late Professor L S Penrose, tests for red cell isoenzymes and plasma protein polymorphisms at the Galton Laboratory, London Univeristy, and satellite counts on chromosome preparations by Dr F R Engmann.

Requests for reprints should be addressed to Dr R M Lewkonia, Department of Medicine, Hammersmith Hospital, London W 12 OHS.

\section{References}

${ }^{1}$ Crabbé, P A, and Heremans, J F, American fournal of Medicine, 1967, 42, 319.

2 Hobbs, J R, Fournal of Clinical Pathology, 1971, 24, suppl 5, p 146.

${ }^{3}$ Bull, D M, and Tomasi, T B, Gastroenterology, 1968, 54, 313.

4 Smith, S M, and Penrose, L S, Annals of Human Genetics, 1955, 19, 273.

5 Asquith, P, Thompson, R A, and Cooke, W T, Lancet, 1969, 2, 129.

6 Bachmann, R, Scandinavian fournal of Clinical and Laboratory Investigations, 1965, 17, 316.

7 Falchuck, Z M, Rogentine, G N, and Strober, W, fournal of Clinical Investigation, 1972, 51, 1602

${ }^{8}$ Lawton, A W, Kincade, P W, and Cooper, M D, Federation Proceedings, $1975,34,33$.

9 Waldmann, T A, et al, Lancet, 1974, 2, 609.

10 Provisor, A J, et al, New England fournal of Medicine, 1975, 293, 62.

11 Crabbé, P A, et al, International Archives of Allergy, 1968, 34, 363.

12 Grundbacher, F J, fournal of Medical Genetics, 1972, 9, 344.

\title{
Changes in immune status in patients undergoing splenectomy for the staging of Hodgkin's disease
}

\author{
B W HANCOCK, LESLEY BRUCE, A MILFORD WARD, JOHN RICHMOND
}

British Medical fournal, 1976, 1, 313-315

\section{Summary}

The immune status of 17 patients with Hodgkin's disease was studied before and after splenectomy (undertaken in staging laparotomy) and during chemotherapy or after radiotherapy. The findings were compared with those in 19 patients not selected for splenectomy. Serum IgA and IgM levels became significantly lower after treatment in the splenectomy group. Cell-mediated immunity was depressed mainly in patients receiving quadruple cytotoxic chemotherapy. Neutrophil function was normal or enhanced and was unchanged after splenectomy and treatment despite changes in neutrophil counts. Three patients who underwent splenectomy suffered fatal septicaemia. These results suggest that humoral immunity is depressed by treatment in patients who have undergone splenectomy, and the benefits of early accurate staging must be weighed against the likelihood of infective complications.

\footnotetext{
University of Sheffield Academic Division of Medicine, Royal Hospital, Sheffield S1 3SR

B W HANCOCK, DCH, MRCP, lecturer

LESLEY BRUCE, HNC, medical laboratory scientist

JOHN RICHMOND, MD, FRCP, professor

Department of Immunology, University of Sheffield, Sheffield A MILFORD WARD, MB, MRCPATH, senior lecturer
}

\begin{abstract}
Introduction
Diagnostic laparotomy with splenectomy is now a recognised procedure in the staging of Hodgkin's disease. In view of the incidence of severe infections after this operation ${ }^{1}$ we have studied serially the immune status of 17 patients with Hodgkin's disease who underwent splenectomy and compared the findings with those in 19 patients seen over the same period who did not undergo splenectomy. Three patients who died with septicaemia after splenectomy and chemotherapy are discussed more fully.
\end{abstract}

\section{Patients}

It is the policy of the lymphoma group in Sheffield to perform diagnostic laparotomy with splenectomy on patients staged clinically (Ann Arbor) as $1 \mathrm{~A}$ and $2 \mathrm{~A}$ (mixed cellularity or lymphocyte depletion histology types) and 1B, 2B, 3A, (all histology types). In the six months from August 1974 to February 197517 such patients underwent operation. During the same period 19 patients did not require laparotomy before starting treatment.

Seven of the patients who underwent splenectomy and nine of the others were subsequently treated with quadruple cytotoxic chemotherapy; the remainder had radiotherapy apart from one patient in each group who had radiotherapy followed by chemotherapy. Immune status was assessed in all patients at presentation, two to four weeks after splenectomy but before treatment and either immediately after radiotherapy or before the fourth course of quadruple chemotherapy.

There were two deaths in the non-splenectomy group from disemination of Hodgkin's disease. In the splenectomy group four patients died, one from dissemination and three from fulminating septicaemia. The last three patients are reported further.

Case 1-A 50-year-old man with stage $4 \mathrm{~B}$ mixed cellularity Hodgkin's disease received six courses of quadruple chemotherapy before being admitted with Pseudomonas aeruginosa septicaemia, which did not respond 